\title{
Interspecific Variations in Duration of Tail Regression in Two Tropical Anurans
}

\author{
Cuckoo Mahapatra ${ }^{1,2}$ and Pravati Kumari Mahapatra' \\ ${ }^{1}$ Cell and Developmental Biology Laboratory, Department of Zoology, Utkal University, Bhubaneswar, Odisha 751 004, India \\ ${ }^{2}$ Department of Zoology, Khallikote Autonomous College, Berhampur, Odisha 760 001, India \\ Correspondence should be addressed to Pravati Kumari Mahapatra; mahapatra.pk.zool@utkaluniversity.ac.in
}

Received 28 August 2015; Revised 9 November 2015; Accepted 11 November 2015

Academic Editor: Victor H. Casco

Copyright (c) 2015 C. Mahapatra and P. K. Mahapatra. This is an open access article distributed under the Creative Commons Attribution License, which permits unrestricted use, distribution, and reproduction in any medium, provided the original work is properly cited.

\begin{abstract}
Anurans breed in an array of habitats and hence employ a variety of evolutionary strategies to adapt to the variable conditions. Particularly, since they undergo a larval phase they develop mechanisms to overcome unfavourable conditions like desiccation, extreme temperatures, and so forth. The anurans, Polypedates maculatus and Duttaphrynus melanostictus, show noticeable variation in the duration of larval period and tadpole tail regression. D. melanostictus breeds throughout the year and hence is subjected to different environmental conditions as compared to P. maculatus which breeds only during the rainy season. Thus, the tadpoles of D. melanostictus have selected to undergo a shorter larval period and duration of tail regression to suit their breeding habits. The present study correlates the interspecific difference in the duration of tail regression with the morphological variations in the tails of the two species. D. melanostictus shortens the duration of larval tail regression by having comparatively larger and more number of melanocytes and a thinner notochord than P. maculatus.
\end{abstract}

\section{Introduction}

Anurans occupy a variety of aquatic environments with different water availabilities which has a profound effect on their life histories. Species breeding in streams and permanent ponds take longer time to complete their larval period as compared to those which breed in temporary and ephemeral ponds, since they are subjected to low desiccation risk. Increased desiccation risks have caused an evolutionary shif $\mathrm{t}$ towards more accelerated development by decreasing body mass at metamorphosis [1]. Higher temperatures have also been reported to lead faster rates of development and shorter time to metamorphosis in anurans [2].

Remarkable variation in the larval period has been observed in two species of tropical anurans, Polypedates maculatus (Rhacophoridae) and Duttaphrynus melanostictus (Bufonidae). P. maculatus lay eggs in the form of foam nest only during the rainy season in temporary as well as permanent ponds. Hence, the tadpoles do not face extreme temperatures and the foam nest prevents the eggs from desiccation as well. Contrastingly, D. melanostictus is a perennial breeder and lays eggs in strings. Eggs are laid in permanent water bodies during winter and summer months but in temporary as well as permanent water bodies in rainy season. The tadpoles are subjected to higher desiccation risks as compared to $P$. maculatus, as the eggs do not have any protective covering except jelly coat. Also they face a variable temperature regime (i.e., minimum $15-19^{\circ} \mathrm{C}$ during winter months, 25 $26^{\circ} \mathrm{C}$ during rainy season, and maximum $35-38^{\circ} \mathrm{C}$ in summer months). In laboratory conditions, at constant temperature and water availability the tadpoles of $P$. maculatus take $40-$ 45 days for completion of larval period and 40-48 hours for complete tail resorption, whereas in D. melanostictus larval period is completed in 22-30 days and tail regression takes 18-24 hours. The toadlets of D. melanostictus having an average snout to tail length (STT) of $8.8 \pm 0.83 \mathrm{~mm}$ are smaller in comparison to the froglets of $P$. maculatus with an average STT of $15.75 \pm 0.95 \mathrm{~mm}$. Also, the tail in tadpoles of $P$. maculatus is longer, constituting $68 \%$ of the total length of the tadpoles as compared to only $55 \%$ in the tadpoles of $D$. melanostictus. Thus it can be assumed that since the tadpoles 
of D. melanostictus are subjected to variable temperature and water availability throughout the year they have adapted to shorter larval period and duration of tail regression even though reared in similar conditions as $P$. maculatus.

In anuran tadpoles metamorphosis begins at Taylor and Kollros (TK) stage XX [3] or Gosner stage 42 [4]. The larval period consists of the premetamorphic (TK stages I-VIII/ Gosner stages 26-33) and prometamorphic periods (TK stages IX-XIX/Gosner stages 34-41). TK stages XX-XXV (Gosner stages 42-46) comprise the climactic stages during which tail is completely resorbed. At stage XX, the forelimb protrudes out and marks the beginning of climax. Tail reduction is visible only at stage XXI. By TK stage XXIII, more than half of the tail regresses and by TK stage XXV, the tail is completely lost. However, the time period for complete resorption of tail (stages $\mathrm{XX}$ to $\mathrm{XXV}$ ) varies from species to species [5].

The tail resorption occurs by apoptosis, which involves death of cells followed by phagocytosis of cell debris by macrophages and other cells $[6,7]$. The involution of tadpole tail is also characterized by the neosynthesis of lysosomal enzymes apparently resulting from the activation of macrophages by some primary autolytic changes in the tail tissue $[8,9]$. An elevation in the level of lysosomal enzymes, during tail regression, has been reported recently in the common Indian tree frog Polypedates maculatus $[9,10]$ and common Asian toad Duttaphrynus melanostictus $[9,11]$.

It has already been established that variations in the larval periods in anurans are regulated by the thyroid hormones [12-14]. Lysosomal enzymes $[9,15]$ and oxidative stress [16] have also been found to be involved in causing interpopulation and interspecific variations in the duration of larval periods and tail regression. But studies on morphofunctional differences have not been attempted earlier. The present study thus aims to evaluate variations in histology and the involvement of lysosomal enzyme cathepsin D in regressing tails that may bring about variations in the duration of tail regression in the above two species of anurans.

\section{Materials and Methods}

2.1. Tadpoles. Foam nests of common Indian tree frog Polypedates maculatus and egg strings of common Asian toad Duttaphrynus melanostictus were collected from around the Utkal University campus in Bhubaneshwar $\left(20^{\circ} 21^{\prime} \mathrm{N} 85^{\circ} 53^{\prime} \mathrm{E}\right)$, Odisha, India, during the month of July in the years 20132015. Tadpoles were reared in the laboratory following the standardized procedure [17]. In laboratory conditions, water availability (i.e., water levels in the rearing tubs) and quality and quantity of food were kept constant. The tadpoles were fed with boiled Amaranthus leaves and boiled egg yolk ad libitum throughout rearing.

2.2. Tail Amputation. The tadpoles were anesthetized with MS 222 (Tricaine methane sulphonate) prior to tail amputation. Tail amputation was done by keeping the specimens laterally on a presterilized porcelain plate. Tails were amputated through the base with the help of a sharp sterilized blade and the whole tail was taken for analysis.

2.3. Chemicals Used. Chemicals used in the study were of the analytical grade. Stains for Mallory's trichrome staining, that is, acid fuchsin, phosphomolybdic acid, aniline blue, and Orange G, were obtained from Ranbaxy, Gurgaon, Haryana, India. Cathepsin D (C-20) goat polyclonal antibody raised against a peptide mapping at the $\mathrm{C}$-terminus of cathepsin $\mathrm{D}$ of human origin was obtained from Santa Cruz Biotechnology, USA. ABC staining system containing antipolyvalent, horseradish peroxidase (HRP)/DAB Kit (Santa Cruz Biotechnology, USA) was used to detect the bound antibodies.

\subsection{Histological Investigation of Tail in TK Stage XXIV/Gosner} Stage 45. For histological analysis, longitudinal sections of tails of stage XXIV tadpoles (Figures 1(a) and 1(b)) were taken. At stage XXIV, tail remains in the form of a stub. The tail remains in this stage for about 6-8 hours in $P$. maculatus and around 4-6 hours in D. melanostictus before it is completely resorbed in stage XXV. For analyses, stage XXIV was again divided into early and late stages. Tail stub for early stages was taken at the commencement of stage XXIV (i.e., tail fins are completely lost) and for late stages, tail was taken just before it is completely resorbed. Thus, early stage XXIV tadpoles had a longer tail stub than the late stages. Following tail amputation, the tail tips were fixed in neutral buffered formalin overnight. Paraffin embedded blocks of the tails were prepared and longitudinal sections of the tail pieces of $5 \mu$ thickness were cut using a rotary microtome machine. The sections were then stained following Mallory's trichrome staining method. All the image analysis procedures were performed by using the ImageJ software, freely available at http://rsb.info.nih.gov/ij/ [18].

2.5. Immunohistochemical Investigation of Tail in TK Stage XXIV/Gosner Stage 45. For immunohistochemical analysis of cathepsin D, late stage XXIV tadpoles were considered. Briefly, deparaffinized and rehydrated sections were pretreated with glycine- $\mathrm{HCl}$ buffer $\left(\mathrm{pH} \mathrm{3.5)}\right.$ at $95^{\circ} \mathrm{C}$ in a water bath for 10 minutes and slides were cooled at room temperature for 20 minutes. The endogenous peroxidase activity was blocked with $1 \% \mathrm{H}_{2} \mathrm{O}_{2}$ for 10 minutes. Then, endogenous biotin was blocked with blocking serum for 1 hour. Sections were incubated overnight with primary antibodies at $4^{\circ} \mathrm{C}$. To visualize the bound primary antibodies, sections were then incubated with biotinylated goat anti-polyvalent secondary antibody for 30 minutes and avidin-biotinylated horse radish peroxidase for 30 minutes. Binding was finally revealed by incubation with diaminobenzidine (DAB) for 10 minutes. Then $1 \%$ haematoxylin counterstain was applied for 1-2 minutes. After washing with $100 \%$ ethanol and clearing with xylene the slides were mounted with DPX. All steps were performed at room temperature unless otherwise specified. Controls were prepared by omitting the primary antibodies and incubating slides with amphibian phosphate buffered saline (APBS) instead. Photographs of clearly stained sections were taken using a compound microscope (Hund, H500) and 


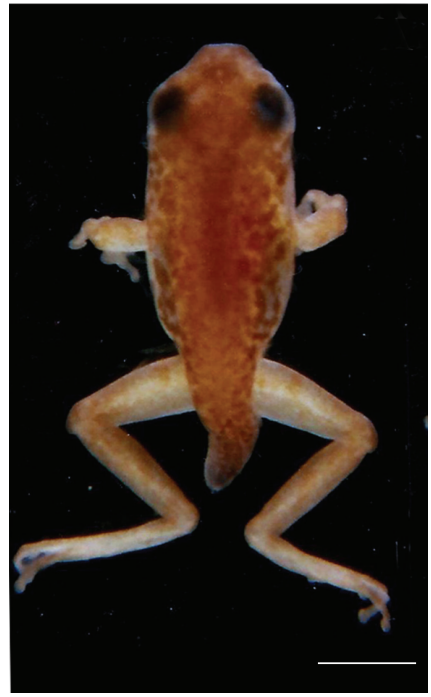

(a)

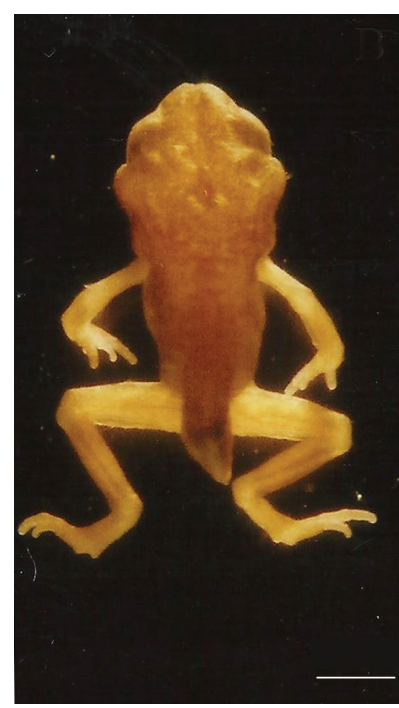

(b)

FIgURE 1: Stage XXIV tadpoles showing tail in form of stub in (a) Polypedates maculatus and (b) Duttaphrynus melanostictus (Bar =1 cm).

Pentax camera and Photoshop 8.0 (Adobe Systems Inc., San Jose, CA, USA) was used to label images and insert scale bars.

\section{Results and Discussion}

3.1. Histological Analysis. In the longitudinal sections of early stage XXIV of P. maculatus (Figure 2(a)) and D. melanostictus (Figure 2(b)), the epidermis (e) was multilayered and wavy. In both species, in the distal part of the tail, wavy notochordal sheath (ns) lined the notochord (n) and the notochordal cells were condensed. In D. melanostictus, a large number of melanocytes $(\mathrm{m})$ were found throughout the tail close to the notochord (Figure 2(b)). But in P. maculatus, melanocytes $(\mathrm{m})$ were present scantily only in the tip of the tail (Figure 2(a)). There was no trace of muscle bundles and degenerating muscle $(\mathrm{dm})$ was present on either side of the notochord in both species. Towards the proximal end, notochord was lined by a straighter notochordal sheath and notochordal cells were prominent. Muscle bundles $(\mathrm{mb})$ were seen in the proximal part of the tail. The epidermis was single layered with a thick basement membrane. The notochord, spinal cord, and blood vessels were observed in patches and were not continuous throughout the tail.

In late stage XXIV of $P$. maculatus (Figure 2(c)) and D. melanostictus (Figure 2(d)) the epidermis (e) was also multilayered and there was no trace of basement membrane. All muscle bundles were degraded and the notochord (n) appeared in patches. The notochordal sheath (ns) had also become thicker and only in the extreme proximal region the notochordal cells retained their shape. In the distal portion, degradation of the notochord (n) was visible with no trace of notochordal sheath and notochordal cells. The degeneration was highest in the distal portion. Melanocytes (m) lined the degenerating notochord in D. melanostictus and the spinal cord was not discernible.
Histology thus revealed bending of the tail structures during degeneration since the tubular structures like notochord, spinal cord, and blood vessels did not appear continuously but in patches. Bending of the notochord during tail regression has been reported by Elinson et al., [19] but bending of spinal cord and blood vessels during tail regression has been reported in the present study for the first time.

The magnified portions of the early stage XXIV tails showed considerable variations in the two species. Quantification of the thickness of the basement membrane and notochord showed that the basement membrane (bm) in $D$. melanostictus was thick and perforated with average thickness of $24.45 \pm 1.4 \mu \mathrm{m}$ but in $P$. maculatus it appeared as a single layer with average thickness of $1.37 \pm 0.31 \mu \mathrm{m}$ (Figures 3(a) and 3(b)). Contrastingly, the notochordal sheath (ns) in $P$. maculatus was thick and perforated with average thickness of $22.37 \pm 2.45 \mu \mathrm{m}$ as compared to D. melanostictus where it appeared as a single layer with average thickness of $4.475 \pm$ $0.66 \mu \mathrm{m}$ (Figures 3(c) and 3(d)).

Variations in the degradation of tail tissues like epidermis and notochord seem to contribute to the variations in the duration of tail regression. Amano et al. [20] have described that the epidermis changes from larval epidermis to a stratified epidermis and finally to a cornified adult epidermis during anuran tail regression. Epidermis (e) in the regressing tails was multilayered or stratified in both the species in the present study and contained cells showing apoptotic morphology (ea) (Figures 3(a) and 3(b)). The numbers of cells showing apoptotic morphology were higher in $D$. melanostictus than in P. maculatus and were in the terminal part of degeneration in D. melanostictus with the formation of apoptotic bodies. Apoptotic bodies are fragments of membrane bound cytoplasm containing organelles, which are formed at the end of an apoptotic process in a cell, and are ultimately taken up by macrophages [21]. Thus, epidermal degradation 


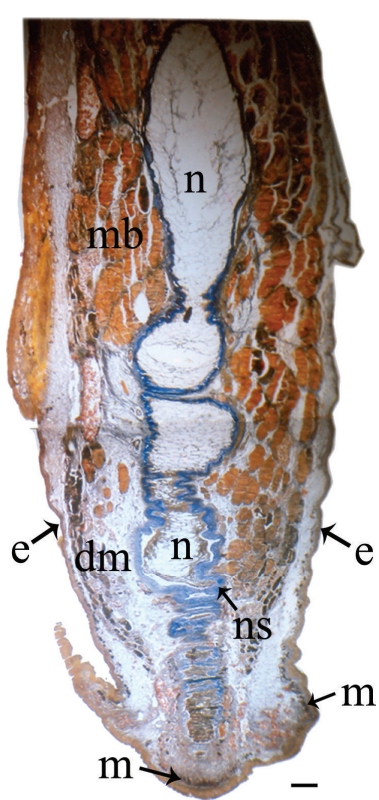

(a)

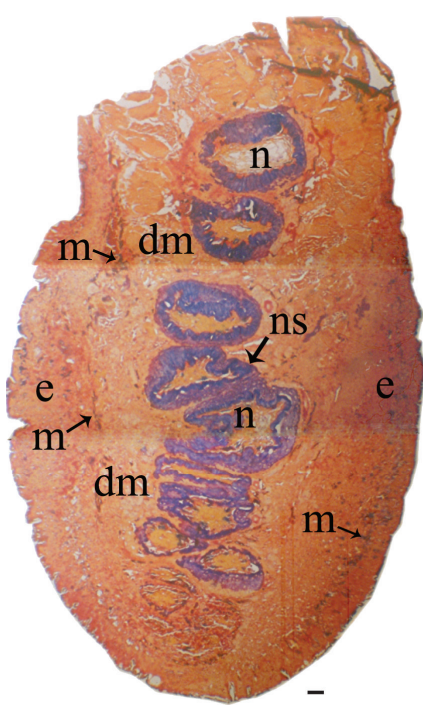

(c)

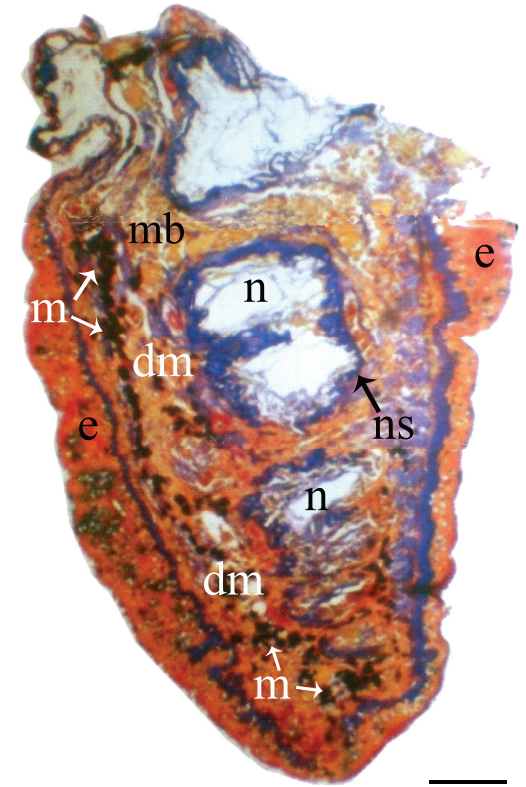

(b)

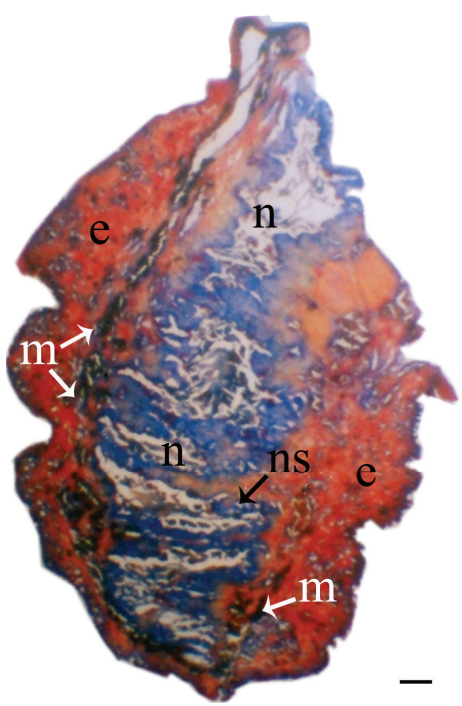

(d)

FIGURE 2: Longitudinal sections of tail at stage XXIV (early and late) of P. maculatus and D. melanostictus. (a) LS through early stage XXIV of $P$. maculatus, (b) LS through early stage XXIV of D. melanostictus, (c) LS through late stage XXIV of $P$. maculatus, and (d) LS through late stage XXIV of $D$. melanostictus (bars $=130 \mu \mathrm{m}$ ); dm: degenerating muscle, e: epidermis, mb: muscle bundle, m: melanocytes, n: notochord, and ns: notochordal sheath.

was higher and was nearing completion in D. melanostictus as compared to $P$. maculatus explaining the faster tail regression in D. melanostictus. Notochordal sheath was thicker in $P$. maculatus as compared to D. melanostictus and evidently would take a longer time for degradation.

Melanocytes $(\mathrm{m})$ in $D$. melanostictus were bigger in size and occupied greater portion of the regressing tail than $P$. maculatus. Quantification of the surface area of melanocytes revealed that, in D. melanostictus, it ranged from 194.41 to $525.93 \mu \mathrm{m}^{2}$ (Figure 3(f)), whereas in P. maculatus it ranged from 114.61 to $404.47 \mu \mathrm{m}^{2}$ (Figure 3(e)). The melanocytes constituted about $4.25 \%$ of the surface area of the section of regressing tail of early stage XXIV of $D$. melanostictus (Figure 2(b)) while in P. maculatus it was found to be only $0.22 \%$ (Figure $2(\mathrm{a})$ ). Similarly, in late stage XXIV tail of D. melanostictus, the melanocytes occupied $5.31 \%$ of the total section of the regressing tail (Figure 2(d)) while in $P$. maculatus the melanocytes occupied only $1.73 \%$ of the total area of the regressing tail (Figure 2(c)). Melanocytes have been associated with the breakdown of skeletal muscle in Clinotarsus curtipes during tail regression [22]. Melanocytes have also been associated with the degradation of epidermis, 


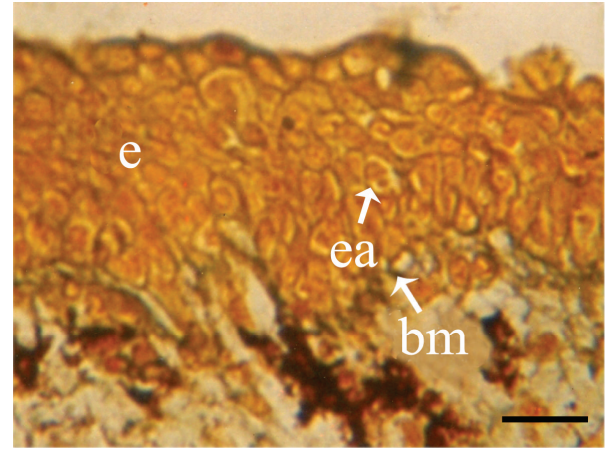

(a)

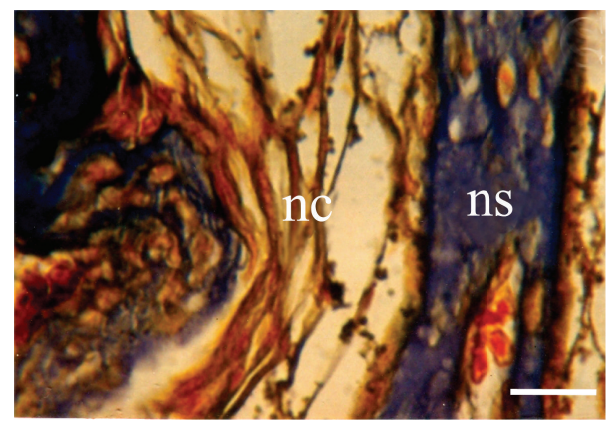

(c)

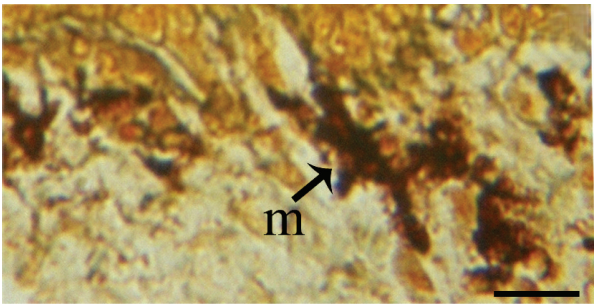

(e)

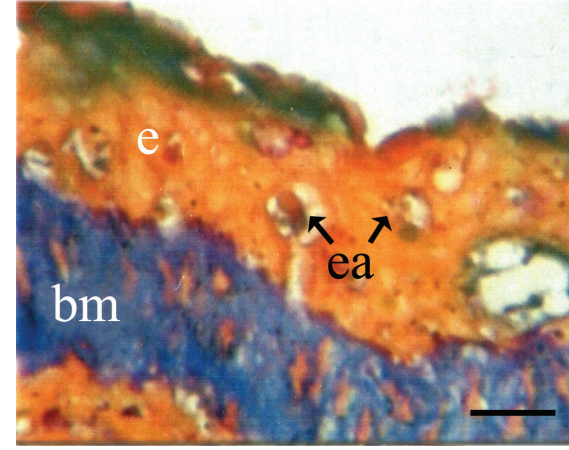

(b)

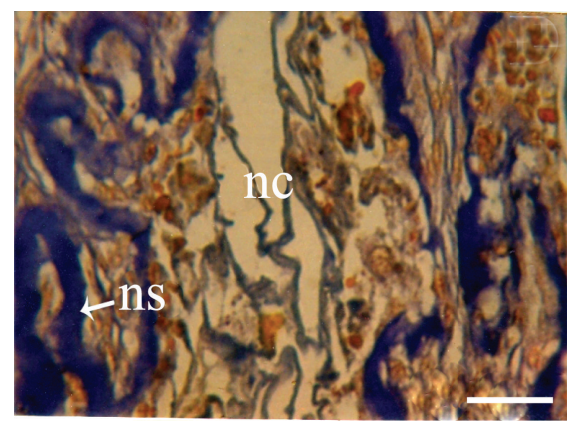

(d)

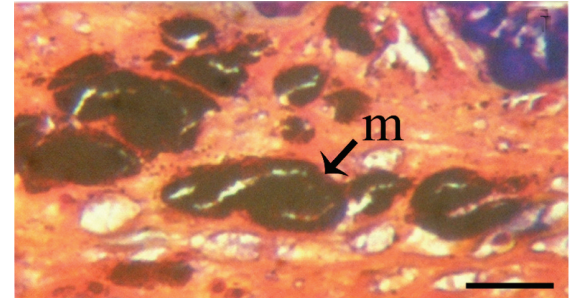

(f)

FIGURE 3: Longitudinal sections of tail at stage XXIV (early) of $P$. maculatus and D. melanostictus showing magnified portions. (a) Epidermis in P. maculatus, (b) epidermis in D. melanostictus, (c) notochord in P. maculatus, (d) notochord in D. melanostictus, (e) melanocytes in P. maculatus, and (f) melanocytes in D. melanostictus (bars $=13 \mu \mathrm{m}$ ); bm: basement membrane, e: epidermis, ea: epidermal cells with apoptotic morphology, m: melanocytes, nc: notochordal cells, and ns: notochordal sheath.

muscle, spinal cord, and blood vessels in the tadpoles of $P$. maculatus and D. melanostictus [10,11]. Melanocytes contain specialized lysosomes called melanosomes that contain a broad array of lysosomal hydrolases [23]. It can be suggested that the bigger and large number of melanocytes in the tails of D. melanostictus may contribute to high levels of lysosomal enzymes and hence faster tail regression. Thus, presence of larger and more melanocytes and thinner notochordal sheath in regressing tails of $D$. melanostictus as compared to $P$. maculatus may explain the rather gradual process of tail resorption in the tadpoles of $P$. maculatus as compared to tadpoles of D. melanostictus.

3.2. Immunohistological Analysis. Release of acid hydrolases from preformed lysosomes is described to be the primary cause of tail atrophy and an increase in activity of lysosomal hydrolase is related to the progressive release of hydrolytic enzymes from preformed lysosomes [24]. Cathepsin D plays an important role in degradation of major tail tissues during tail resorption in tadpoles of $P$. maculatus and D. melanostictus $[10,11]$. The present study thus compared the localization of cathepsin $\mathrm{D}$ in the regressing tail tissues of $P$. maculatus and D. melanostictus to ascertain the involvement of cathepsin D in causing variations in the duration of tail regression.

The tail in stage XXIV (late) showed strong positive signals for cathepsin D in both the species. Melanocytes (m) were distributed throughout the tails near the degrading tail tissues. Melanocytes (m) were distributed below the epidermis in both P. maculatus and D. melanostictus but in $D$. melanostictus they were closer to the notochord (Figures 4(a) and 4(b)). Cathepsin D localization could be visualised in the degrading tissues like epidermis, muscle, and inner layers of notochord. In the epidermis (e), apoptotic cells appeared as large cells with pyknotic nuclei and retraction from the cell membrane (ea). The epidermis appeared as a single thick layer embedded with large spaces containing cathepsin D 


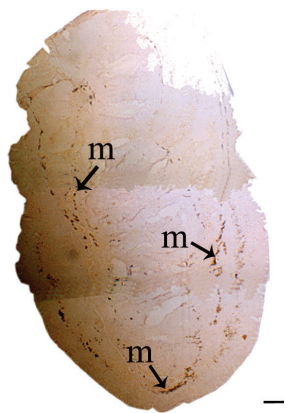

(a)

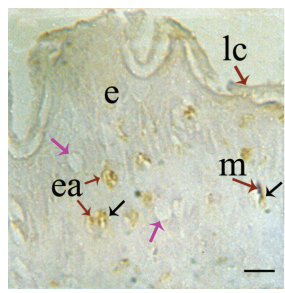

(c)

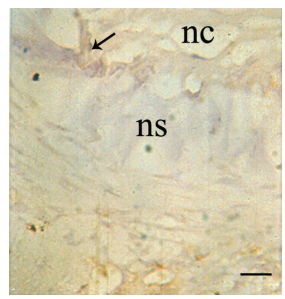

(e)

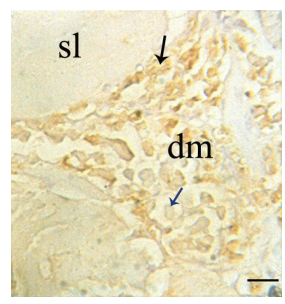

(g)

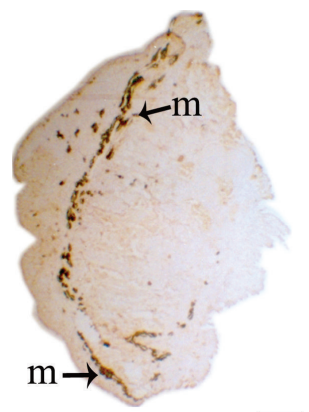

(b)

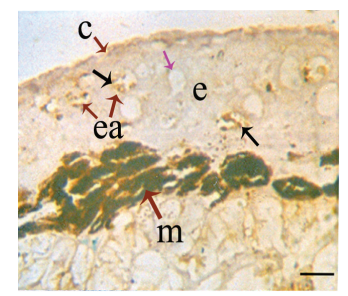

(d)

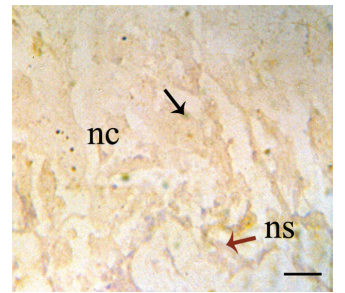

(f)

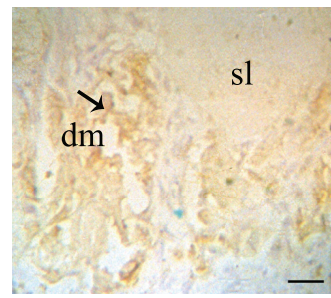

(h)

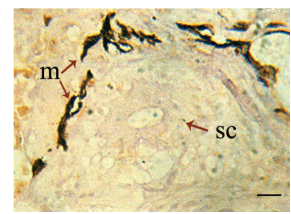

(i)

FIgURE 4: Longitudinal sections of tail at stage XXIV (late) of P. maculatus and D. melanostictus showing localization of cathepsin D. (a) LS through whole tail of $P$. maculatus, (b) LS through whole tail of D. melanostictus, (c) epidermis in P. maculatus, (d) epidermis in D. melanostictus, (e) notochord in P. maculatus, (f) notochord in D. melanostictus, (g) muscle in P. maculatus, (h) muscle in D. melanostictus, and (i) spinal cord in P. maculatus (bars in (a) and (b) $=130 \mu \mathrm{m},(\mathrm{c})-(\mathrm{i})=13 \mu \mathrm{m}$ ) (black arrows indicate cathepsin D positive tissues, blue arrow indicates degraded tissue which is not cathepsin D positive, and pink arrows indicate empty spaces); c: cuticle, dm: degenerating muscle, e: epidermis, ea: epidermal cells with apoptotic morphology, lc: loose cuticle, m: melanocytes, nc: notochordal cells, ns: notochordal sheath, sl: sarcolytes, and sc: spinal cord. 
positive material (Figures 4(c) and 4(d)). The inner layer of notochordal sheath (ns) and the membrane of notochordal cells (nc) also stained for cathepsin D (Figures 4(e) and 4(f)). Degrading myofibrils $(\mathrm{dm})$ stained positive for cathepsin D and were surrounded by sarcolytes (sl) (Figures $4(\mathrm{~g})$ and 4(h)). The spinal cords in both the species were negative for cathepsin D. Melanocytes (m) were also found around the nerve cords of $P$. maculatus (Figure 4(i)). Mahapatra et al. [9] have identified cathepsin D as an important enzyme in causing variations in the duration of tail regression. However, the present study did not record any differences in localization of cathepsin D in the regressing tissues of both the species although large number of melanocytes may contribute higher amounts of cathepsin D and faster tail regression in D. melanostictus.

\section{Conclusion}

The ability to adjust the duration of larval period to the local habitat quality may have played a major role in allowing different populations and even species to adapt to widely divergent environments. Anurans like D. melanostictus enhance their flexibility to breed throughout the year by adapting to a shorter larval period and duration of tail regression as compared to $P$. maculatus which breeds only during rainy season. Therefore, the duration of larval period and tail regression in tadpoles of D. melanostictus is always shorter than $P$. maculatus although they are reared in similar conditions. Present study reports for first time that faster rate of tail regression in $D$. melanostictus can be associated to the morphological differences in the tail tissues. Since D. melanostictus tadpoles are subjected to varying climatic conditions they have acquired specializations like larger melanocytes and thinner notochord to reduce the duration of tail regression.

\section{Conflict of Interests}

The authors declare that there is no conflict of interests regarding the publication of this paper.

\section{Acknowledgments}

Financial assistance from Department of Science and Technology, Government of India (PURSE grant), to P. G. Department of Zoology, Utkal University, is gratefully acknowledged. Cuckoo Mahapatra would like to thank the University Grants Commission for a Senior Research Fellowship.

\section{References}

[1] A. Richter-Boix, M. Tejedo, and E. L. Rezende, "Evolution and plasticity of anuran larval development in response to desiccation: a comparative analysis," Ecology and Evolution, vol. 1, no. 1, pp. 15-25, 2011.

[2] G. R. Ultsch, D. E. Bradford, and J. Freda, "Physiology: coping with the environment," in Tadpoles: The Biology of Anuran Larvae, R. W. McDiarmid and R. Altig, Eds., pp. 189-214, University of Chicago Press, Chicago, Ill, USA, 1999.
[3] A. C. Taylor and J. J. Kollros, "Stages in the normal development of Rana pipiens larvae," The Anatomical Record, vol. 94, no. 1, pp. 7-23, 1946.

[4] K. L. Gosner, "A simplified table for staging anuran embryos and larvae with notes on identification," Herpetologica, vol. 16, no. 3, pp. 183-190, 1960.

[5] W. E. Duellman and L. Trueb, Biology of Amphibians, McGrawHill, New York, NY, USA, 1985.

[6] J. F. Kerr, B. Harmon, and J. Searle, "An electron microscope study of cell deletion in anuran tadpole tail during spontaneous metamorphosis with special reference to apoptosis of striated muscle fibres," Journal of Cell Science, vol. 14, pp. 571-585, 1974.

[7] L. I. Gilbert and E. Freiden, Metamorphosis: A problem in Developmental Biology, Plenum, New York, NY, USA, 2nd edition, 1981.

[8] P. K. Mahapatra, P. Mohanty-Hejmadi, and G. B. N. Chainy, "Changes in oxidative stress parameters and acid phosphatase activity in the pre-regressing and regressing tail of Indian jumping frog Polypedates maculatus (Anura, Rhacophoridae)," Comparative Biochemistry and Physiology C: Toxicology \& Pharmacology, vol. 130, no. 3, pp. 281-288, 2001.

[9] P. K. Mahapatra, S. N. Jamdar, C. Mahapatra, S. K. Dutta, and P. Harikumar, "Interspecies variation in activity of lysosomal cathepsins during tail regression in the tadpoles of three sympatric anurans," Russian Journal of Herpetology, vol. 19, no. 3, pp. 232-238, 2012.

[10] C. Mahapatra and P. K. Mahapatra, "Involvement of cathepsin $\mathrm{D}$ during tail regression in tadpoles of the common Indian tree frog, Polypedates maculatus (Anura: Rhacophoridae)," Acta Histochemica, vol. 113, no. 7, pp. 756-762, 2011.

[11] C. Mahapatra and P. K. Mahapatra, "Immunohistochemical localization of cathepsin $\mathrm{D}$ and a possible role of melanocytes during tail resorption in tadpoles of a tropical toad," Journal of Morphology, vol. 273, no. 7, pp. 791-800, 2012.

[12] R. J. Denver, N. Mirhadi, and M. Phillips, "Adaptive plasticity in amphibian metamorphosis: response of Scaphiopus hammondii tadpoles to habitat desiccation," Ecology, vol. 79, no. 6, pp. 18591872,1998

[13] Y.-B. Shi, Amphibian Metamorphosis: From Morphology to Molecular Biology, Wiley-Liss, New York, NY, USA, 2000.

[14] C. S. Rose, "Integrating ecology and developmental biology to explain the timing of frog metamorphosis," Trends in Ecology and Evolution, vol. 20, no. 3, pp. 129-135, 2005.

[15] C. Mahapatra, S. K. Dutta, and P. K. Mahapatra, "Interpopulational differences in activity of acid phosphatase in the tails of the tadpoles of Duttaphrynus melanostictus (Anura: Bufonidae)," Russian Journal of Herpetology, vol. 18, no. 2, pp. 105-110, 2011.

[16] I. Gomez-Mestre, S. Kulkarni, and D. R. Buchholz, "Mechanisms and consequences of developmental acceleration in tadpoles responding to pond drying," PLoS ONE, vol. 8, no. 12, Article ID e84266, 2013.

[17] P. Mohanty-Hejmadi, "Care and management of amphibian embryos," Prakruti-Utkal University Journal of Science, vol. 11, pp. 81-87, 1977.

[18] C. A. Schneider, W. S. Rasband, and K. W. Eliceiri, "NIH Image to ImageJ: 25 years of image analysis," Nature Methods, vol. 9, no. 7, pp. 671-675, 2012.

[19] R. P. Elinson, B. Remo, and D. D. Brown, "Novel structural elements identified during tail resorption in Xenopus laevis metamorphosis: lessons from tailed frogs," Developmental Biology, vol. 215, no. 2, pp. 243-252, 1999. 
[20] T. Amano, H. Kawabata, and K. Yoshizato, "Characterization of metamorphic changes in anuran larval epidermis using lectins as probes," Development Growth and Differentiation, vol. 37, no. 2, pp. 211-220, 1995.

[21] G. Häcker, “The morphology of apoptosis," Cell and Tissue Research, vol. 301, no. 1, pp. 5-17, 2000.

[22] L. Divya, R. S. Beyo, P. Sreejith, M. A. Akbarsha, and O. V. Oommen, "Skeletal muscle-melanocyte association during tadpole tail resorption in a tropical frog, Clinotarsus curtipes Jerdon (Anura, Ranoidea)," Zoology, vol. 113, no. 3, pp. 175-183, 2010.

[23] S. Diment, M. Eidelman, G. M. Rodriguez, and S. J. Orlow, "Lysosomal hydrolases are present in melanosomes and are elevated in melanizing cells," The Journal of Biological Chemistry, vol. 270, no. 9, pp. 4213-4215, 1995.

[24] R. Weber, The Biochemistry of Animal Development, Academic Press, New York, NY, USA, 1968. 

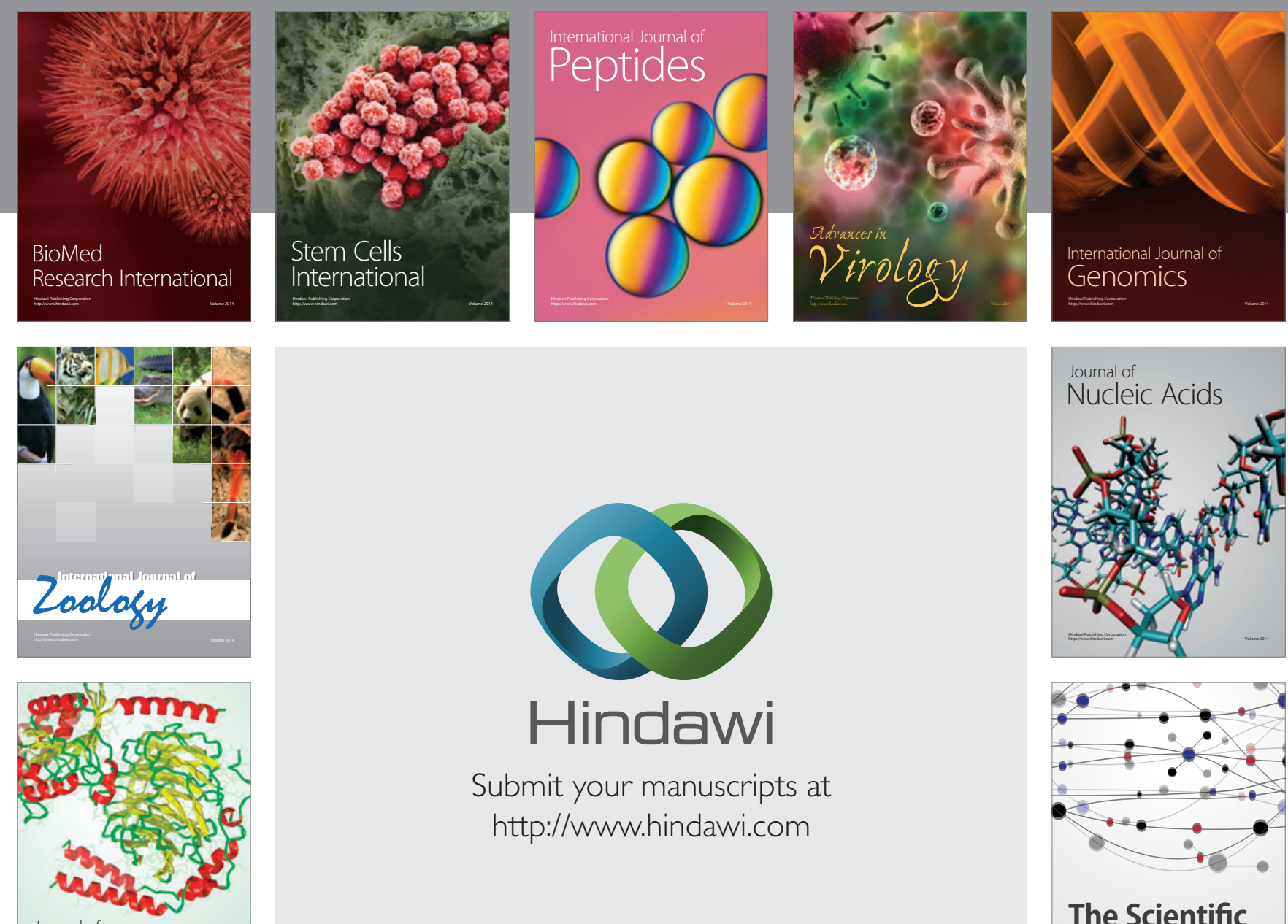

Submit your manuscripts at

http://www.hindawi.com

Journal of
Signal Transduction
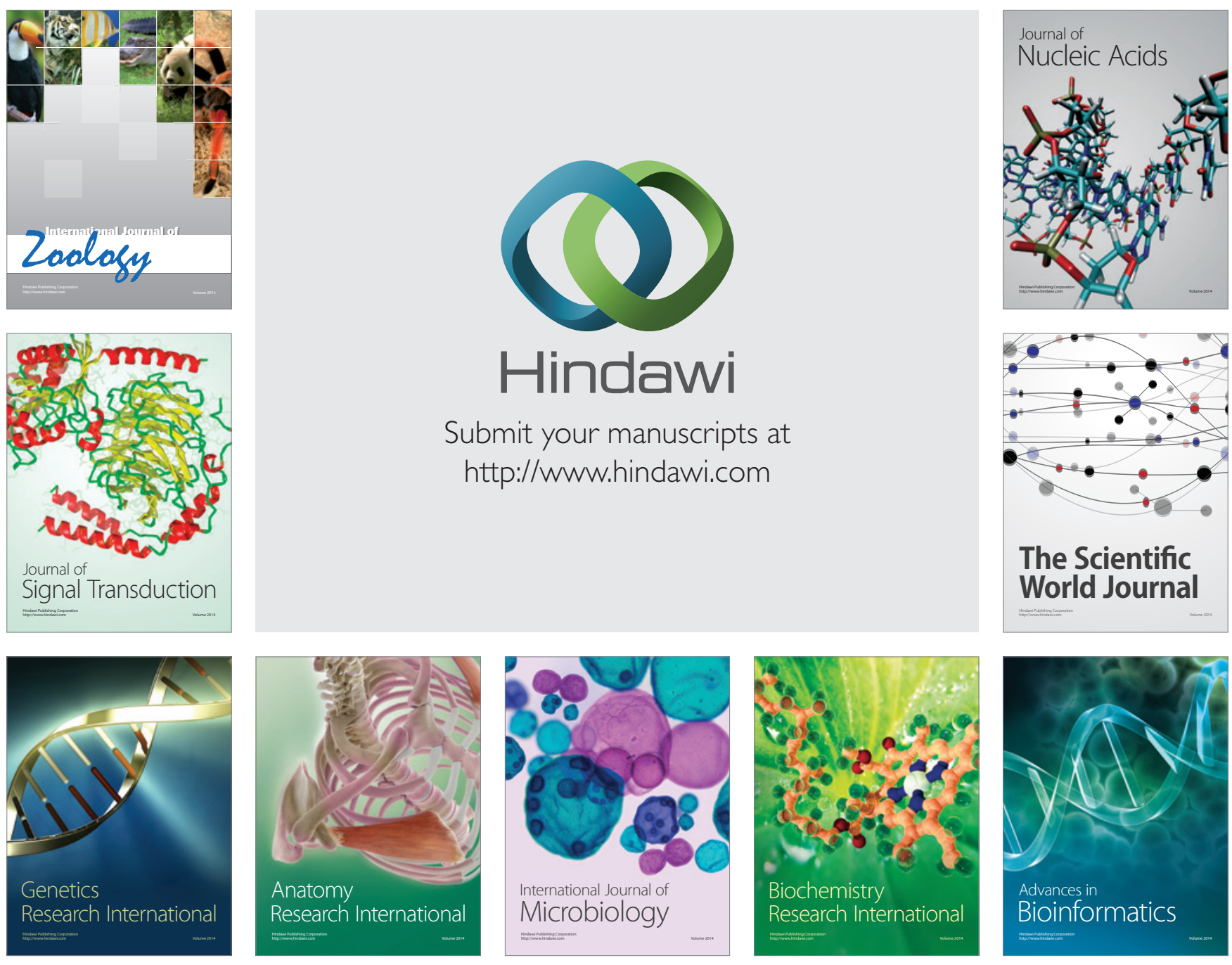

The Scientific World Journal
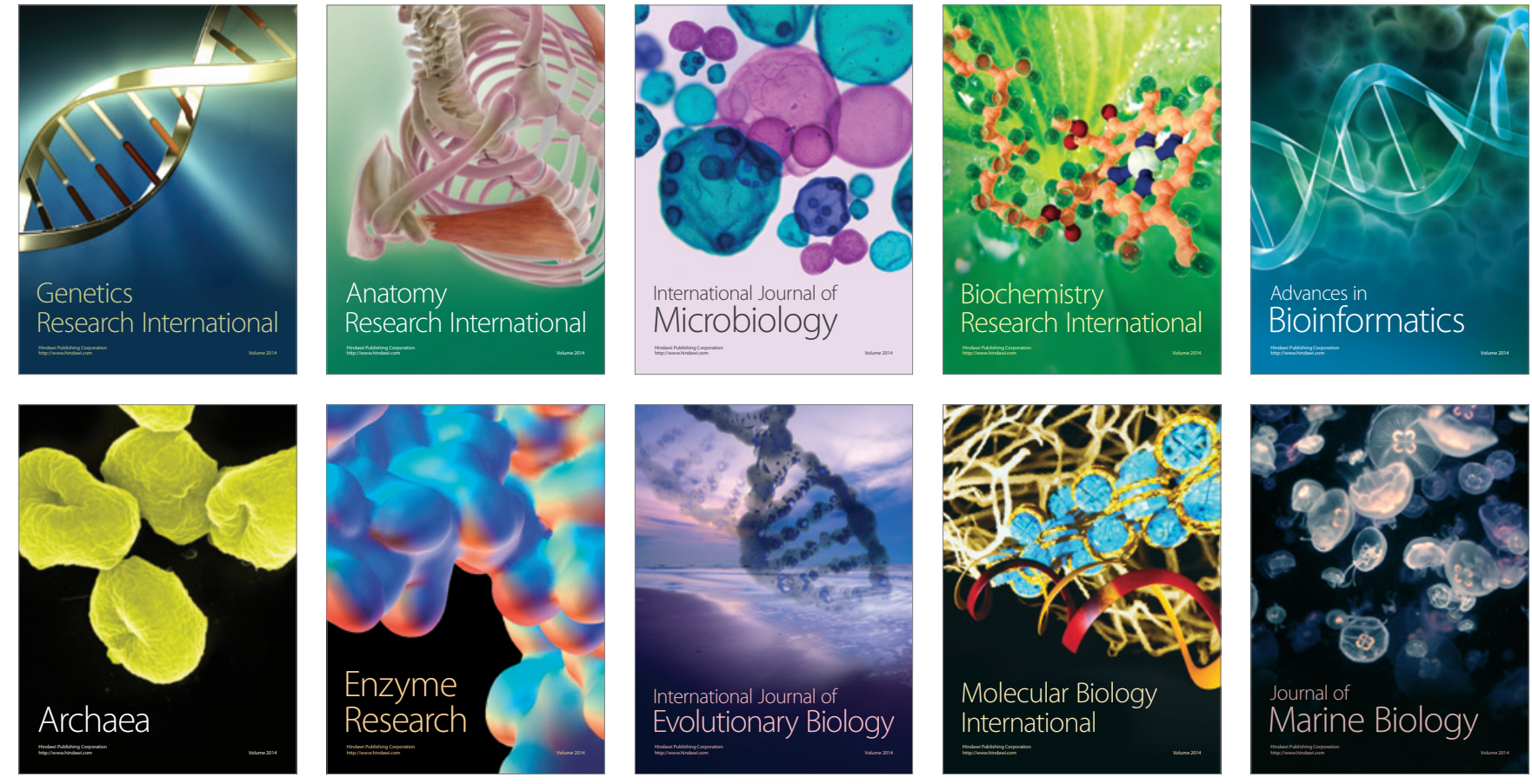